\title{
Multiplicity of periodic bouncing solutions for generalized impact Hamiltonian systems
}

Delong Huang ${ }^{1}$ and Fei Guo ${ }^{*}$

*Correspondence:

guofei79@tju.edu.cn

${ }^{1}$ School of Mathematics, Tianjin

University, Tianjin, China

\begin{abstract}
Applying the Generalized Nonsmooth Saddle Point Theorem, we obtain multiple nontrivial periodic bouncing solutions for systems $\ddot{x}=f(t, x)$ with new conditions. In particular, we generalize the collision axis from $x=0$ to the axis $x=a$, where $a$ is an arbitrary constant.

MSC: $34 \mathrm{C} 25 ; 49 J 35 ; 70 \mathrm{H} 05 ; 74 \mathrm{M} 20 ; 74 \mathrm{G} 35$

Keywords: Generalized Nonsmooth Saddle Point Theorem; Periodic bouncing solution; Impact Hamiltonian systems; Multiplicity
\end{abstract}

\section{Introduction}

Consider the following Hamiltonian system with an obstacle, that is,

$$
\ddot{x}=f(t, x), \quad t \in \mathbf{R} \backslash W,
$$

associated with the conditions

$$
\begin{cases}\dot{x}\left(t^{-}\right)=-\dot{x}\left(t^{+}\right), & t \in W, \\ x(t) \geq a, & \forall t \in \mathbf{R}, \\ x(t)=x(t+T), & \forall t \in \mathbf{R},\end{cases}
$$

where $a$ is a constant, $W=\{t \in \mathbf{R} \mid x(t)=a\}$, and $f: \mathbf{R} \times[a,+\infty) \rightarrow \mathbf{R}$ is continuous and $T$-periodic in $t$.

Definition 1.1 (see [11]) Let $x: \mathbf{R} \rightarrow \mathbf{R}$ be continuous map. Then $x$ is a nonrivial $T$ periodic bouncing solution of system (1.1) with collision axis at $x=a$ if it satisfies (1.1)(1.2) and

(1) the set $W$ is nonempty and discrete,

(2) there exists at least one $t_{0} \in W$ such that $\dot{x}\left(t_{0}^{-}\right) \neq 0$.

We call systems, having solutions as in Definition 1.1, impact Hamiltonian systems. When $a=0$, the bouncing periodic solutions of (1.1) have been discussed by some scholars in recent years. To the best of the authors' knowledge, Jiang (see [7]) first proposed a variational method to consider the bouncing periodic solutions of equation (1.1), and

(c) The Author(s) 2019. This article is distributed under the terms of the Creative Commons Attribution 4.0 International License (http://creativecommons.org/licenses/by/4.0/), which permits unrestricted use, distribution, and reproduction in any medium, provided you give appropriate credit to the original author(s) and the source, provide a link to the Creative Commons license, and indicate if changes were made. 
obtained the existence of bouncing solutions for impact Hamiltonian systems under a classical superquadratic condition. Then Ding (see [3]) considered the existence of subharmonic bouncing solutions for system (1.1) with sublinear conditions. In 2017, Nie (see [9]) first proved a Generalized Nonsmooth Saddle Point Theorem, which is applied to impact Hamiltonian systems, then obtained nontrivial $k T$-periodic bouncing solutions for system (1.1) with another sublinear condition.

Different from the papers [3, 7] and [9], we focus on the nontrivial $k T$-periodic bouncing solutions for system (1.1) with a new condition separating whether or not $a$ is equal to 0 . The idea comes from the papers [7] and [9].

In this paper, we suppose that $\dot{x}\left(t_{i}^{-}\right) \leq 0$, furthermore, $\dot{x}\left(t_{i}^{-}\right)<0$ if $t_{i}$ is a real impact time. To ensure that there exists at least one real impact time, paper [9] tells us that we must give the following condition:

(B) The inequality $f(t, x) \leq 0$ holds for every $t \in[0, T]$ and $x \geq a$; moreover, $\lim _{x \rightarrow+\infty} f(t, x)=-\infty$ or $\limsup _{x \rightarrow+\infty} f(t, x)<0$ holds for every $t \in[0, T]$.

Now, we list our main result of periodic bouncing solution as follows:

Set $\Gamma=\{h \in C([0,+\infty) ;[0,+\infty)) \mid h$ satisfies (h1)-(h4) $\}$, where

(h1) $h(s) \leq h(t)+C$, for a certain constant $C>0$ and $s, t \in[0,+\infty)$ with $s \leq t$,

(h2) $h(s+t) \leq C^{*}(h(s)+h(t))$, for a certain constant $C^{*} \geq 0$ and $\forall s, t \in[0,+\infty)$,

(h3) $\operatorname{th}(t)-2 H(t) \rightarrow-\infty$, as $t \rightarrow+\infty$,

(h4) $\frac{H(t)}{t^{2}} \rightarrow 0$, as $t \rightarrow+\infty$, and $H(t):=\int_{0}^{t} h(s) \mathrm{d} s$. Since $h(t)=\sqrt{t}$ can be in $\Gamma$, we get $\Gamma \neq \emptyset$.

We suppose that function $f$ satisfies the following conditions:

(f) There exist $T$-periodic functions $\gamma, g \in L^{1}([0, T] ;(0,+\infty))$ and function $h \in \Gamma$ such that

$$
|f(t,|x|+a)| \leq \gamma(t) h(|x|)+g(t), \quad \forall x \in \mathbf{R} \text { and } t \in[0, T] .
$$

(F1) Function $h \in \Gamma$ in condition (f) satisfies

$$
\limsup _{|x| \rightarrow+\infty} \frac{1}{H(|x|)} \int_{0}^{T} F(t,|x|+a) \mathrm{d} t<0
$$

where $F(t, x)=\int_{a}^{x} f(t, s) \mathrm{d} s(x \geq a)$.

(F2) Function $f(t, x)$ is differentiable for a.e. $t \in[0, T]$ and there exists a constant $\sigma>0$ such that

$$
\left|\frac{\partial F(t, x)}{\partial t}\right| \leq-\sigma F(t, x), \quad \text { a.e. } t \in[0, T] \text { and } x \in[a,+\infty)
$$

Theorem 1.1 Suppose function $f$ satisfies conditions (B), (f), (F1) and (F2). Then system (1.1) possesses nontrivial $k T$-periodic bouncing solutions $u_{k}$ for any sufficiently large integer $k$. Furthermore, $\left\|u_{k}\right\|_{L^{\infty}} \rightarrow+\infty$ as $k \rightarrow+\infty$.

In this paper, we generalize the collision axis from $x=0$ to the axis $x=a$, so our conditions generalize those in [9]. The main difficulty of this paper is checking whether the corresponding functional is locally Lipschitz and finding the range of the generalized gradients of the above functional. Therefore, we first prove Lemma 2.1 in Sect. 2. 
System (1.1) with condition (1.2) is equivalent to the system

$$
\ddot{x}=f(t, x+a), \quad t \in \mathbf{R} \backslash W^{1},
$$

associated with the conditions

$$
\begin{cases}\dot{x}\left(t^{-}\right)=-\dot{x}\left(t^{+}\right), & t \in W^{1}, \\ x(t) \geq 0, & \forall t \in \mathbf{R}, \\ x(t)=x(t+T), & \forall t \in \mathbf{R},\end{cases}
$$

where $W^{1}=\{t \in \mathbf{R} \mid x(t)=0\}$.

Based on similar proofs in paper [7], we can conclude that if $x: \mathbf{R} \rightarrow \mathbf{R}$ is a $T$-periodic solution with isolated zeros of

$$
\ddot{x}=f(t,|x|+a) \operatorname{sgn}(x), \quad t \in \mathbf{R} \backslash W^{1},
$$

then $|x|$ is a nontrivial $T$-periodic bouncing solution of system (1.4) with collision axis $x=0$, and vice versa.

\section{Preliminaries}

Some concepts and conclusions about the Clarke generalized gradient can be found in [2] and [1], so we omit them.

Definition 2.1 (see [5]) Function $\varphi$ satisfies the nonsmooth (PS) condition if every sequence $\left\{x_{n}\right\} \subset E$, such that $\left\{\varphi\left(x_{n}\right)\right\}$ is bounded and $\lambda\left(x_{n}\right) \rightarrow 0$ for $n \rightarrow \infty$, has a strongly convergent subsequence, where $\lambda(x)=\inf _{x^{*} \in \partial \varphi(x)}\left\|x^{*}\right\|_{E^{*}}, E^{*}$ is the dual space of $E$, and $\partial \varphi(x)$ denotes the Clarke generalized gradient of $\varphi$.

Theorem 2.1 (Generalized Nonsmooth Saddle Point Theorem, see [9]) Let E be a real Banach space, and $E=V \oplus X$ with $V \neq\{\mathbf{0}\}$ and $\operatorname{dim} V<+\infty$. Suppose that functional $\varphi$ satisfies the nonsmooth (PS) condition, and for some $x_{0} \in X$, there exists a constant $r>0$ such that $\max _{v \in V \cap \partial B_{r}} \varphi\left(v+x_{0}\right)<\inf _{x \in X} \varphi(x)$. If $c$ can be characterized as $c=\inf _{\chi \in \Gamma_{1}} \max _{v \in V \cap \bar{B}_{r}} \varphi\left(\chi\left(v+x_{0}\right)\right)$, then $c$ is a critical value of $\varphi$, where $\Gamma_{1}=\{\chi \in$ $C\left(V \cap \bar{B}_{r}+x_{0}, E\right) \mid \chi\left(v+x_{0}\right)=v+x_{0}$, if $\left.v \in V \cap \partial B_{r}\right\}$ and $B_{r}=\{x \in E \mid\|x\|<r\}$. Furthermore, we have $c \geq \inf _{x \in X} \varphi(x)$.

Set

$$
H_{k T}^{1}=\left\{\begin{array}{l|l}
x:[0, k T] \rightarrow \mathbf{R} & \begin{array}{l}
x(t) \text { is absolutely continuous, } \\
x(0)=x(k T), \dot{x} \in L^{2}([0, k T], \mathbf{R})
\end{array}
\end{array}\right\},
$$

in which $k \in \mathbf{N}^{*}$, then $H_{k T}^{1}$ is a Hilbert space with the norm defined by $\|x\|=\left[\int_{0}^{k T}\left(|\dot{x}(t)|^{2}+\right.\right.$ $\left.\left.|x(t)|^{2}\right) \mathrm{d} t\right]^{\frac{1}{2}}$. For $x \in H_{k T}^{1}$, let $\bar{x}=\frac{1}{k T} \int_{0}^{k T} x(t) \mathrm{d} t$ and $\tilde{x}(t)=x(t)-\bar{x}$. The book [8] tells us the following Wirtinger's inequality:

$$
\int_{0}^{k T}|\tilde{x}(t)|^{2} \mathrm{~d} t \leq \frac{k^{2} T^{2}}{4 \pi^{2}} \int_{0}^{k T}|\dot{x}(t)|^{2} \mathrm{~d} t
$$


and Sobolev's inequality

$$
\|\tilde{x}\|_{L^{\infty}}^{2} \leq \frac{k T}{12} \int_{0}^{k T}|\dot{x}(t)|^{2} \mathrm{~d} t
$$

Set $\|x\|_{0}=\left(|\bar{x}|^{2}+\int_{0}^{k T}|\dot{x}(t)|^{2} \mathrm{~d} t\right)^{\frac{1}{2}}$, then the norm $\|\cdot\|_{0}$ is equivalent to $\|\cdot\|$ (see [8]). Indeed, $H_{k T}^{1}$ has the decomposition $H_{k T}^{1}=\mathbf{R} \oplus \tilde{H}_{k T}^{1}$, where $\tilde{H}_{k T}^{1}=\left\{x \in H_{k T}^{1} \mid \bar{x}=0\right\}$. Let $J_{k}(x)=$ $\int_{0}^{k T} F(t,|x(t)|+a) \mathrm{d} t, \forall x \in H_{k T}^{1}$, and let $\varphi_{k}(x)=\frac{1}{2} \int_{0}^{k T}|\dot{x}(t)|^{2} \mathrm{~d} t+J_{k}(x), \forall x \in H_{k T}^{1}$.

Lemma 2.1 Iff satisfies condition (f), then functional $J_{k}$ is locally Lipschitz on $H_{k T}^{1}$ and

$$
\partial J_{k}(x) \subseteq\left[f^{-}(t,|x(t)|+a), f^{+}(t,|x(t)|+a)\right] \text { a.e. } t \in[0, k T],
$$

where

$$
\begin{aligned}
& f^{-}(t,|s|+a)=\min \left\{\lim _{u \rightarrow s^{-}} f(t,|s|+a) \operatorname{sgn}(u), \lim _{u \rightarrow s^{+}} f(t,|s|+a) \operatorname{sgn}(u)\right\}, \\
& f^{+}(t,|s|+a)=\max \left\{\lim _{u \rightarrow s^{-}} f(t,|s|+a) \operatorname{sgn}(u), \lim _{u \rightarrow s^{+}} f(t,|s|+a) \operatorname{sgn}(u)\right\} .
\end{aligned}
$$

Proof The main idea comes from [1]. Considering the functional

$$
J_{k}(x)=\int_{0}^{k T} \mathrm{~d} t \int_{0}^{|x(t)|} f(t, s+a) \mathrm{d} s
$$

we have

$$
\begin{aligned}
\left|J_{k}(u)-J_{k}(v)\right| & \leq \int_{0}^{k T}\left|\int_{|v(t)|}^{|u(t)|}[\gamma(t) h(s)+g(t)] \mathrm{d} s\right| \mathrm{d} t \\
& \leq \int_{0}^{k T}|\gamma(t) \widetilde{h}(t)+g(t)||| u(t)|-| v(t)|| \mathrm{d} t \\
& \leq\left(\int_{0}^{k T}(\gamma(t) \widetilde{h}(t)+g(t))^{2} \mathrm{~d} t\right)^{\frac{1}{2}}\left(\int_{0}^{k T}(u(t)-v(t))^{2} \mathrm{~d} t\right)^{\frac{1}{2}} \\
& =K\|u-v\|_{L^{2}} \leq K \cdot C\|u-v\|_{H_{k T}^{1}},
\end{aligned}
$$

where $\widetilde{h}(t)=\max \{h(s) \mid s$ is between $|v(t)|$ and $|u(t)|\}, K:=\left(\int_{0}^{k T}(\gamma(t) \widetilde{h}(t)+g(t))^{2} \mathrm{~d} t\right)^{\frac{1}{2}}>0$, $C$ is embedding constant, (2.4) means that $J_{k}$ is locally Lipschitz continuous, so [1] tells us that the generalized gradients of $F$ at $x+a$ do exist. The generalized gradients of $F$ at $x+a$ $(x>0)$ are denoted by $\partial F(t, x+a)=\left.\partial_{v} F^{0}(t, x+a ; v)\right|_{v=0}$, where $\partial_{v} F^{0}(t, x+a ; v)$ denotes the subdifferential in $v$ of $F^{0}(t, x+a ; v)$, and $F^{0}(t, x+a ; v)=\limsup _{h \rightarrow 0, \mu \rightarrow 0^{+}} \frac{1}{\mu} \int_{x+h}^{x+h+\mu v} f(t, s+$ a) $\mathrm{d} s$. Then we have

$$
F^{0}(t, x+a ; v) \leq \begin{cases}v \lim _{\sigma \rightarrow 0^{+}} \min _{s \in[x-\sigma, x+\sigma]} f(t, s+a)=f^{-}(t, x+a) v, & \text { if } v \leq 0 \\ v \lim _{\sigma \rightarrow 0^{+}} \max _{s \in[x-\sigma, x+\sigma]} f(t, s+a)=f^{+}(t, x+a) v, & \text { if } v \geq 0 .\end{cases}
$$


By (2.5) and results in [1], we have $\partial F(t, x+a) \subseteq\left[f^{-}(t, x+a), f^{+}(t, x+a)\right]$. By definition, $\exists h_{i} \in H_{k T}^{1}, h_{i} \rightarrow 0(i \rightarrow+\infty)$ in $H_{k T}^{1}$ such that

$$
J_{k}^{0}(x ; v)=\limsup _{i \rightarrow+\infty, \mu \rightarrow 0^{+}} \frac{1}{\mu} \int_{0}^{k T} \mathrm{~d} t \int_{h_{i}(t)}^{h_{i}(t)+\mu v(t)} f(t, s+|x(t)|+a) \mathrm{d} s,
$$

so using the results of [1], we have

$$
\begin{aligned}
J_{k}^{0}(x ; v) \leq & \int_{0}^{k T} F^{0}(t,|x(t)|+a ; v) \mathrm{d} t \\
= & \int_{0}^{k T} \max \{\omega \cdot v(t) \mid \omega \in \partial F(t,|x(t)|+a)\} \mathrm{d} t \\
\leq & \int_{v(t)>0} v(t) f^{+}(t,|x(t)|+a) \mathrm{d} t \\
& +\int_{v(t)<0} v(t) f^{-}(t,|x(t)|+a) \mathrm{d} t .
\end{aligned}
$$

If $\omega_{0} \in \partial J_{k}(x)$, we are going to prove

$$
f^{-}(t,|x(t)|+a) \leq \omega_{0}(t) \leq f^{+}(t,|x(t)|+a), \quad \forall t \in \mathbf{R} .
$$

Otherwise, there would be a set $E_{0}$, for example, on which

$$
f^{-}(t,|x(t)|+a)>\omega_{0}(t), \quad \forall t \in E_{0}
$$

Due to results in [1],

$$
\partial \varphi\left(x_{0}\right)=\left\{w \in E^{*} \mid\langle w, v\rangle \leq \varphi^{0}\left(x_{0} ; v\right), \forall v \in E\right\}
$$

Let $v_{0}(t)=-\chi_{E_{0}}(t)$, the characteristic function of $E_{0}$. Then, from (2.8), we have

$$
J_{k}^{0}\left(x ; v_{0}\right) \geq \int_{0}^{k T} \omega_{0}(t) \cdot v_{0}(t) \mathrm{d} t=-\int_{E_{0}} \omega_{0}(t) \mathrm{d} t .
$$

From the definition of $v_{0}(t)$, we have

$$
\begin{aligned}
& -\int_{E_{0}} f^{-}(t,|x(t)|+a) \mathrm{d} t \\
& \quad=\int_{v_{0}(t)>0} v_{0}(t) \cdot f^{+}(t,|x(t)|+a) \mathrm{d} t+\int_{v_{0}(t)<0} v_{0}(t) \cdot f^{-}(t,|x(t)|+a) \mathrm{d} t .
\end{aligned}
$$

Equations (2.10), (2.6) and (2.9) imply that

$$
-\int_{E_{0}} f^{-}(t,|x(t)|+a) \mathrm{d} t \geq-\int_{E_{0}} \omega_{0}(t) \mathrm{d} t
$$

which contradicts (2.7). Similarly, we can get $\omega_{0}(t) \leq f^{+}(t,|x(t)|+a)$. 
Remark 2.1 For $w \in \partial J_{k}(x)$, there is a function $\varpi$ with $\varpi(t) \in\left[f^{-}(t,|x(t)|+a), f^{+}(t,|x(t)|+\right.$ a)] a.e. $t \in[0, k T]$ such that $\langle w, v\rangle=\int_{0}^{k T} \varpi(t) v(t) \mathrm{d} t$ holds for all $v \in H_{k T}^{1}$.

Remark 2.2 When $s \neq 0$, we have $f^{-}(t,|s|+a)=f^{+}(t,|s|+a)=f(t,|s|+a) \operatorname{sgn}(s)$. If $s=0$ and $f$ satisfies condition (B), then we have $f^{-}(t,|s|+a)=f(t,|s|+a)$ and $f^{+}(t,|s|+a)=-f(t,|s|+a)$.

Lemma 2.2 If $x_{k} \in H_{k T}^{1}$ is a critical point of $\varphi_{k}$ on $H_{k T}^{1}$, then $x_{k}$ is a periodic solution of equation (1.6), and vice versa.

Proof The main idea comes from [7]. Lemma 2.1 means that $\varphi_{k}$ is locally Lipschitz continuous, so $\partial \varphi_{k}$ do exist. Let $x$ be a critical point of $\varphi_{k}$ in $H_{k T}^{1}$, in other words, $\mathbf{0} \in \partial \varphi_{k}$. By Remark 2.1, there is a function $g$ with $g(t) \in\left[f^{-}(t,|x(t)|+a), f^{+}(t,|x(t)|+a)\right]$ such that

$$
\int_{0}^{k T}(\dot{x}(t) \dot{v}(t)+g(t) v(t)) \mathrm{d} t=0, \quad \forall v \in H_{k T}^{1}
$$

which shows that the generalized second order derivative $\ddot{x}$ exists and $x$ satisfies

$$
\int_{0}^{k T}(-\ddot{x}(t)+g(t)) v(t) \mathrm{d} t=0, \quad \forall v \in H_{k T}^{1}
$$

that is,

$$
\ddot{x}=g(t) \text { a.e. } t \in \mathbf{R} .
$$

We need to prove that $g(t)=f(t,|x|+a) \operatorname{sgn}(x)$. Let $T_{0}=\{t \in[0, k T] \mid x(t)=0\}$ and $T_{1}=$ $\{t \in[0, k T] \mid x(t) \neq 0\}$. Remark 2.2 tells us that $g(t)=f^{-}(t,|x|+a)=f^{+}(t,|x|+a)=f(t,|x|+$ a) $\operatorname{sgn}(x)$ for $t \in T_{1}$. Using [6, Lemma 7.7], we obtain $\ddot{x}=\dot{x}=0$ for a.e. $t \in T_{0}$, and draw a conclusion from (2.11) that $g(t)=0=f(t,|x|+a) \operatorname{sgn}(x)$ holds for a.e. $t \in T_{0}$. Thus we complete the proof.

\section{Bouncing solutions for second order Hamiltonian systems}

Lemma 3.1 Functional $\varphi_{k}$ satisfies the nonsmooth (PS) condition iff satisfies (f) and (F1).

Proof The main idea comes from [9]. Let $\left\{x_{n}\right\} \in H_{k T}^{1}$ be a nonsmooth (PS) sequence, that is, $\left\{\varphi\left(x_{n}\right)\right\}$ is bounded and $\lambda\left(x_{n}\right) \rightarrow 0$ as $n \rightarrow \infty$. According to Remark 2.1 and the definition of $\lambda\left(x_{n}\right)$, for each $n \in \mathbf{N}^{*}$, there exist functions $\varpi_{n} \in \partial J_{k}\left(x_{n}\right)$ and $x_{n}^{*} \in \partial \varphi_{k}\left(x_{n}\right)$ with $\left\|x_{n}^{*}\right\|_{E^{*}} \rightarrow 0$ as $n \rightarrow \infty$ such that

$$
\left\langle x_{n}^{*}, v\right\rangle=\int_{0}^{k T} \dot{x}_{n}(t) \dot{v}(t) \mathrm{d} t+\int_{0}^{k T} \varpi_{n}(t) v(t) \mathrm{d} t, \quad \forall v \in H_{k T}^{1} .
$$

For $x_{n} \in H_{k T}^{1}=\mathbf{R} \oplus \tilde{H}_{k T}^{1}$, we write $x_{n}$ as $x_{n}(t)=\bar{x}_{n}+\tilde{x}_{n}(t)$ for all $t \in[0, k T]$, where $\bar{x}_{n} \in \mathbf{R}$, $\tilde{x}_{n}(t) \in \tilde{H}_{k T}^{1}$. It follows from (2.3), (1.3), (h1), and (h2) that

$$
\begin{aligned}
\left|\varpi_{n}(t)\right| & \leq\left|f\left(t,\left|x_{n}(t)\right|+a\right)\right| \\
& \leq \gamma(t) h\left(\left|x_{n}(t)\right|\right)+g(t)
\end{aligned}
$$




$$
\begin{aligned}
& \leq \gamma(t)\left[h\left(\left|\bar{x}_{n}\right|+\left|\tilde{x}_{n}(t)\right|\right)+C\right]+g(t) \\
& \leq C^{*} \gamma(t) h\left(\left|\bar{x}_{n}\right|\right)+C^{*} \gamma(t) h\left(\left|\tilde{x}_{n}(t)\right|\right)+C \gamma(t)+g(t) .
\end{aligned}
$$

Using (3.2), (h1), Young inequality, [9, (1) of Lemma 2.3] and (2.2), we have

$$
\begin{aligned}
& \left|\int_{0}^{k T} \varpi_{n}(t) \tilde{x}_{n}(t) \mathrm{d} t\right| \\
& \leq \int_{0}^{k T}\left|\varpi_{n}(t)\right|\left|\tilde{x}_{n}(t)\right| \mathrm{d} t \\
& \leq \int_{0}^{k T}\left[C^{*} \gamma(t) h\left(\left|\bar{x}_{n}\right|\right)+C^{*} \gamma(t) h\left(\left|\tilde{x}_{n}(t)\right|\right)+C \gamma(t)+g(t)\right]\left|\tilde{x}_{n}(t)\right| \mathrm{d} t \\
& \leq C^{*}\left\|\tilde{x}_{n}\right\|_{L^{\infty}} h\left(\left|\bar{x}_{n}\right|\right) \int_{0}^{k T} \gamma(t) \mathrm{d} t+C^{*}\left[h\left(\left\|\tilde{x}_{n}\right\|_{L^{\infty}}\right)+C\right]\left\|\tilde{x}_{n}\right\|_{L^{\infty}} \int_{0}^{k T} \gamma(t) \mathrm{d} t \\
& +C\left\|\tilde{x}_{n}\right\|_{L^{\infty}} \int_{0}^{k T} \gamma(t) \mathrm{d} t+\left\|\tilde{x}_{n}\right\|_{L^{\infty}} \int_{0}^{k T} g(t) \mathrm{d} t \\
& \leq C^{*}\left[\frac{3}{C^{*} k T}\left\|\tilde{x}_{n}\right\|_{L^{\infty}}^{2}+\frac{C^{*} k T}{3} h^{2}\left(\left|\bar{x}_{n}\right|\right)\left(\int_{0}^{k T} \gamma(t) \mathrm{d} t\right)^{2}\right] \\
& +C^{*}\left(\varepsilon\left\|\tilde{x}_{n}\right\|_{L^{\infty}}+C_{\varepsilon}+C\right)\left\|\tilde{x}_{n}\right\|_{L^{\infty}} \int_{0}^{k T} \gamma(t) \mathrm{d} t \\
& +C\left\|\tilde{x}_{n}\right\|_{L^{\infty}} \int_{0}^{k T} \gamma(t) \mathrm{d} t+\left\|\tilde{x}_{n}\right\|_{L^{\infty}} \int_{0}^{k T} g(t) \mathrm{d} t \\
& \leq \frac{1}{4} \int_{0}^{k T}\left|\dot{x}_{n}(t)\right|^{2} \mathrm{~d} t+\frac{C^{*^{2}} k T}{3} h^{2}\left(\left|\bar{x}_{n}\right|\right)\left(\int_{0}^{k T} \gamma(t) \mathrm{d} t\right)^{2} \\
& +\frac{\varepsilon C^{*} k T}{12} \int_{0}^{k T}\left|\dot{x}_{n}(t)\right|^{2} \mathrm{~d} t \int_{0}^{k T} \gamma(t) \mathrm{d} t \\
& +\left(C^{*} C_{\varepsilon}+C^{*} C+C\right)\left(\frac{k T}{12} \int_{0}^{k T}\left|\dot{x}_{n}(t)\right|^{2} \mathrm{~d} t\right)^{\frac{1}{2}} \int_{0}^{k T} \gamma(t) \mathrm{d} t \\
& +\left(\frac{k T}{12} \int_{0}^{k T}\left|\dot{x}_{n}(t)\right|^{2} \mathrm{~d} t\right)^{\frac{1}{2}} \int_{0}^{k T} g(t) \mathrm{d} t \\
& \leq\left(\frac{1}{4}+\varepsilon C_{1}\right) \int_{0}^{k T}\left|\dot{x}_{n}(t)\right|^{2} \mathrm{~d} t+C_{2, \varepsilon}\left(\int_{0}^{k T}\left|\dot{x}_{n}(t)\right|^{2} \mathrm{~d} t\right)^{\frac{1}{2}}+C_{3} h^{2}\left(\left|\bar{x}_{n}\right|\right),
\end{aligned}
$$

where $C_{1}=\frac{C^{*} k T}{12} \int_{0}^{k T} \gamma(t) \mathrm{d} t>0, C_{2, \varepsilon}=\sqrt{\frac{k T}{12}}\left(C^{*} C_{\varepsilon}+C^{*} C+C\right) \int_{0}^{k T} \gamma(t) \mathrm{d} t+\sqrt{\frac{k T}{12}} \int_{0}^{k T} g(t) \mathrm{d} t>$ 0 and $C_{3}=\frac{C^{*} k T}{3}\left(\int_{0}^{k T} \gamma(t) \mathrm{d} t\right)^{2}>0$. By (3.1) and (3.3), for $n$ large enough, we have

$$
\begin{aligned}
\left\|\tilde{x}_{n}\right\| \geq & \left\langle x_{n}^{*}, \tilde{x}_{n}\right\rangle=\int_{0}^{k T}\left|\dot{x}_{n}(t)\right|^{2} \mathrm{~d} t+\int_{0}^{k T} \varpi_{n}(t) \tilde{x}_{n}(t) \mathrm{d} t \\
\geq & \left(\frac{3}{4}-\varepsilon C_{1}\right) \int_{0}^{k T}\left|\dot{x}_{n}(t)\right|^{2} \mathrm{~d} t-C_{2, \varepsilon}\left(\int_{0}^{k T}\left|\dot{x}_{n}(t)\right|^{2} \mathrm{~d} t\right)^{\frac{1}{2}} \\
& -C_{3} h^{2}\left(\left|\bar{x}_{n}\right|\right) .
\end{aligned}
$$


On the other hand, (2.1) implies that

$$
\left\|\tilde{x}_{n}\right\| \leq\left(1+\frac{k^{2} T^{2}}{4 \pi^{2}}\right)^{\frac{1}{2}}\left(\int_{0}^{k T}\left|\dot{x}_{n}(t)\right|^{2} \mathrm{~d} t\right)^{\frac{1}{2}} \quad \forall n \in \mathbf{N}^{*}
$$

Let $\varepsilon>0$ be small enough such that $\frac{3}{4}-\varepsilon C_{1}>0$. Using the property of the parabola, then (3.4) and (3.5) mean that there exist two constants $C_{4}, C_{5}>0$ such that

$$
\left(\int_{0}^{k T}\left|\dot{x}_{n}(t)\right|^{2} \mathrm{~d} t\right)^{\frac{1}{2}} \leq C_{4} h\left(\left|\bar{x}_{n}\right|\right)+C_{5} \quad \text { for } n \text { large enough. }
$$

By the mean value theorem for a locally Lipschitz functional (see [5]), there exists $z_{n} \in$ $\left\{(1-s) x_{n}+s \bar{x}_{n} \mid 0 \leq s \leq 1\right\}$ and $z_{n}^{*} \in \partial J_{k}\left(z_{n}\right)$ such that

$$
\int_{0}^{k T} F\left(t,\left|x_{n}(t)\right|+a\right) \mathrm{d} t-\int_{0}^{k T} F\left(t,\left|\bar{x}_{n}\right|+a\right) \mathrm{d} t=\left\langle z_{n}^{*}, x_{n}-\bar{x}_{n}\right\rangle
$$

By Lemma 2.1, there exists a function $\varpi_{z_{n}}(t) \in\left[f^{-}\left(t,\left|z_{n}(t)\right|+a\right), f^{+}\left(t,\left|z_{n}(t)\right|+a\right)\right]$ such that

$$
\left\langle z_{n}^{*}, x_{n}-\bar{x}_{n}\right\rangle=\int_{0}^{k T}\left(\varpi_{z_{n}}(t), x_{n}(t)-\bar{x}_{n}\right) \mathrm{d} t
$$

In the same way as in the computation of (3.3) with $\varepsilon=1$, together with (3.7) and (3.8), we have

$$
\begin{aligned}
& \int_{0}^{k T} F\left(t,\left|x_{n}(t)\right|+a\right) \mathrm{d} t-\int_{0}^{k T} F\left(t,\left|\bar{x}_{n}\right|+a\right) \mathrm{d} t \\
& \quad=\int_{0}^{k T}\left(\varpi_{z_{n}}(t), x_{n}(t)-\bar{x}_{n}\right) \mathrm{d} t \\
& \quad \leq \int_{0}^{k T}\left|f\left(t,\left|z_{n}(t)\right|+a\right)\right|\left|x_{n}(t)-\bar{x}_{n}\right| \mathrm{d} t \\
& \quad=\int_{0}^{k T}\left|f\left(t,\left|\bar{x}_{n}+(1-s) \tilde{x}_{n}(t)\right|+a\right)\right|\left|\tilde{x}_{n}(t)\right| \mathrm{d} t \\
& \quad \leq \int_{0}^{k T}\left[C^{*} \gamma(t) h\left(\left|\bar{x}_{n}\right|\right)+C^{*} \gamma(t) h\left(\left|\tilde{x}_{n}(t)\right|\right)+C \gamma(t)+g(t)\right]\left|\tilde{x}_{n}(t)\right| \mathrm{d} t
\end{aligned}
$$

which is similar to (3.3), and we have

$$
\begin{aligned}
& \int_{0}^{k T} F\left(t,\left|x_{n}(t)\right|+a\right) \mathrm{d} t-\int_{0}^{k T} F\left(t,\left|\bar{x}_{n}\right|+a\right) \mathrm{d} t \\
& \quad \leq\left(\frac{1}{4}+C_{1}\right) \int_{0}^{k T}\left|\dot{x}_{n}(t)\right|^{2} \mathrm{~d} t+C_{2}\left(\int_{0}^{k T}\left|\dot{x}_{n}(t)\right|^{2} \mathrm{~d} t\right)^{\frac{1}{2}}+C_{3} h^{2}\left(\left|\bar{x}_{n}\right|\right)
\end{aligned}
$$

By (3.9), (3.6), [9, Lemma 2.3] and (F1), one has

$$
\begin{aligned}
\varphi_{k}\left(x_{n}\right)= & \frac{1}{2} \int_{0}^{k T}\left|\dot{x}_{n}(t)\right|^{2} \mathrm{~d} t+\int_{0}^{k T}\left[F\left(t,\left|x_{n}(t)\right|+a\right)-F\left(t,\left|\bar{x}_{n}\right|+a\right)\right] \mathrm{d} t \\
& +\int_{0}^{k T} F\left(t,\left|\bar{x}_{n}\right|+a\right) \mathrm{d} t
\end{aligned}
$$




$$
\begin{aligned}
\leq & \left(\frac{3}{4}+C_{1}\right) \int_{0}^{k T}\left|\dot{x}_{n}(t)\right|^{2} \mathrm{~d} t+C_{2}\left(\int_{0}^{k T}\left|\dot{x}_{n}(t)\right|^{2} \mathrm{~d} t\right)^{\frac{1}{2}}+C_{3} h^{2}\left(\left|\bar{x}_{n}\right|\right) \\
& +\int_{0}^{k T} F\left(t,\left|\bar{x}_{n}\right|+a\right) \mathrm{d} t \\
\leq & \left(\frac{3}{4}+C_{1}\right)\left[C_{4} h\left(\left|\bar{x}_{n}\right|\right)+C_{5}\right]^{2}+C_{2}\left[C_{4} h\left(\left|\bar{x}_{n}\right|\right)+C_{5}\right]+C_{3} h^{2}\left(\left|\bar{x}_{n}\right|\right) \\
& +\int_{0}^{k T} F\left(t,\left|\bar{x}_{n}\right|+a\right) \mathrm{d} t \\
\leq & C_{6} h^{2}\left(\left|\bar{x}_{n}\right|\right)+C_{7} h\left(\left|\bar{x}_{n}\right|\right)+\int_{0}^{k T} F\left(t,\left|\bar{x}_{n}\right|+a\right) \mathrm{d} t+C_{8} \\
= & H\left(\left|\bar{x}_{n}\right|\right)\left[C_{6} \frac{h^{2}\left(\left|\bar{x}_{n}\right|\right)}{H\left(\left|\bar{x}_{n}\right|\right)}+C_{7} \frac{h\left(\left|\bar{x}_{n}\right|\right)}{H\left(\left|\bar{x}_{n}\right|\right)}+\frac{\int_{0}^{k T} F\left(t,\left|\bar{x}_{n}\right|+a\right) \mathrm{d} t}{H\left(\left|\bar{x}_{n}\right|\right)}\right]+C_{8} \\
\rightarrow & -\infty, \quad \text { as }\left|\bar{x}_{n}\right| \rightarrow+\infty
\end{aligned}
$$

(where constants $C_{6}>0, C_{7}>0, C_{8}>0$ ) which contradicts the boundedness of $\left\{\varphi_{k}\left(x_{n}\right)\right\}$, thus $\left\{\bar{x}_{n}\right\}$ is bounded and, together with (3.6), one has that $\left\{\left\|x_{n}\right\|_{0}\right\}$ is bounded. Then, by the equivalence of the two norms, $\left\{\left\|x_{n}\right\|\right\}$ is bounded.

Next, we verify that $\left\{x_{n}\right\}$ has a strongly convergent subsequence. The main idea comes from [9] and [10].

Suppose $x_{n} \rightarrow x$ in $H_{k T}^{1}$, then $x_{n} \rightarrow x$ in $C([0, k T] ; \mathbf{R})$. The results in [1] imply that $\partial \varphi_{k}\left(x_{n}\right)$ is weak*-compact, and the set-valued mapping $x \rightarrow \partial \varphi_{k}(x)$ is upper semicontinuous, so, according to [9], we get $x_{n} \rightarrow x$ in $H_{k T}^{1}$, and hence $\varphi_{k}$ satisfies the nonsmooth (PS) condition.

Lemma 3.2 For every $k \in \mathbf{N}^{*}$, functional $\varphi_{k}(x) \rightarrow+\infty$ as $\|x\| \rightarrow+\infty$ in $\tilde{H}_{k T}^{1}$, iff satisfies condition (f).

Proof The main idea comes from [9].

For every $x \in \tilde{H}_{k T}^{1}$, by (f), (h1), of [9, (1) of Lemma 2.3] and (2.2), we have

$$
\begin{aligned}
\left|\int_{0}^{k T} F(t,|x(t)|+a) \mathrm{d} t\right| & \leq \int_{0}^{k T} \mathrm{~d} t \int_{0}^{|x(t)|}|f(t, s+a)| \mathrm{d} s \\
& \leq \int_{0}^{k T} \mathrm{~d} t \int_{0}^{|x(t)|}[\gamma(t) h(s)+g(t)] \mathrm{d} s \\
& \leq \int_{0}^{k T}\left[\gamma(t) h\left(\|x\|_{L^{\infty}}\right)+C \gamma(t)+g(t)\right](|x(t)|) \mathrm{d} t \\
& \leq \varepsilon C_{9}\|x\|_{L^{\infty}}^{2}+C_{10, \varepsilon}\|x\|_{L^{\infty}} \\
& \leq \varepsilon C_{11} \int_{0}^{k T}|\dot{x}(t)|^{2} \mathrm{~d} t+C_{12, \varepsilon}\left(\int_{0}^{k T}|\dot{x}(t)|^{2} \mathrm{~d} t\right)^{\frac{1}{2}},
\end{aligned}
$$

where $C_{10, \varepsilon}, C_{12, \varepsilon}, C_{13, \varepsilon}>0$ hold for any $\varepsilon>0$, and $C_{9}, C_{11}>0$. Then (3.10) implies

$$
\varphi_{k}(x) \geq\left(\frac{1}{2}-\varepsilon C_{11}\right) \int_{0}^{k T}|\dot{x}(t)|^{2} \mathrm{~d} t-C_{12, \varepsilon}\left(\int_{0}^{k T}|\dot{x}(t)|^{2} \mathrm{~d} t\right)^{\frac{1}{2}} \quad \forall x \in \tilde{H}_{k T}^{1} .
$$


Choose $\varepsilon>0$ small enough such that $\frac{1}{2}-\varepsilon C_{11}>0$. In $\tilde{H}_{k T}^{1},\|x\| \rightarrow+\infty$ if and only if $\left(\int_{0}^{k T}|\dot{x}(t)|^{2} \mathrm{~d} t\right)^{\frac{1}{2}} \rightarrow+\infty$, and then (3.11) implies that $\varphi_{k}(x) \rightarrow+\infty$ as $\|x\| \rightarrow+\infty$.

Lemma 3.3 For every $k \in \mathbf{N}^{*}$, we have $\varphi_{k}\left(x+e_{k}\right) \rightarrow-\infty$ as $|x| \rightarrow+\infty$ in $\mathbf{R} \subseteq H_{k T}^{1}$, where $e_{k}(t)=k \cos \left(\frac{2 \pi t}{k T}\right) \in \tilde{H}_{k T}^{1}$, iff satisfies conditions (f) and (F1).

Proof The main idea comes from [9]. Using (3.9), (F1) and [9, Lemma 2.3], we have

$$
\begin{aligned}
\varphi_{k}\left(x+e_{k}\right)= & \frac{2 k \pi^{2}}{T}+\int_{0}^{k T}\left[F\left(t,\left|x+e_{k}(t)\right|+a\right)-F(t,|x|+a)\right] \mathrm{d} t \\
& +\int_{0}^{k T} F(t,|x|+a) \mathrm{d} t \\
\leq & \frac{2 k \pi^{2}}{T}+\left(\frac{1}{4}+C_{1}\right) \int_{0}^{k T}\left|\dot{e}_{k}(t)\right|^{2} \mathrm{~d} t+C_{2}\left(\int_{0}^{k T}\left|\dot{e}_{k}(t)\right|^{2} \mathrm{~d} t\right)^{\frac{1}{2}} \\
& +C_{3} h^{2}(|x|)+\int_{0}^{k T} F(t,|x|+a) \mathrm{d} t \\
= & \frac{2 k \pi^{2}}{T}+\left(\frac{1}{4}+C_{1}\right) \frac{2 k \pi^{2}}{T}+C_{2} \pi \sqrt{\frac{2 k}{T}} \\
& +H(|x|)\left[C_{3} \frac{h^{2}(|x|)}{H(|x|)}+\frac{\int_{0}^{k T} F(t,|x|+a) \mathrm{d} t}{H(|x|)}\right] \\
\rightarrow & -\infty, \quad \text { as }|x| \rightarrow+\infty, x \in \mathbf{R} .
\end{aligned}
$$

Proposition 3.1 If conditions (f) and (F1) hold, then there exists a constant $r_{0}>0$ large enough such that functional $\varphi_{k}$ has at least one critical value $c_{k}$ characterized by

$$
c_{k}=\inf _{\chi \in \Gamma_{2}} \max _{x \in\left[-r_{0}, r_{0}\right]} \varphi_{k}\left(\chi\left(x+e_{k}\right)\right),
$$

where $\Gamma_{2}=\left\{\chi \in C\left(\left[-r_{0}, r_{0}\right]+e_{k}, E\right) \mid \chi\left(e_{k} \pm r_{0}\right)=e_{k} \pm r_{0}\right\}$. Furthermore, for $\forall k \in \mathbf{N}^{*}$, then

$$
-\infty<\inf _{\tilde{H}_{k T}^{1}} \varphi_{k} \leq c_{k} \leq \sup _{x \in \mathbf{R}} \varphi_{k}\left(x+e_{k}\right)
$$

Proof Set $V=\mathbf{R}$ and $X=\tilde{H}_{k T}^{1}$, Lemmas 3.2 and 3.3 tell us that there exists a constant $r_{0}>0$ large enough such that

$$
\max _{x \in V \cap \partial B_{r_{0}}} \varphi_{k}\left(x+e_{k}\right)<\inf _{x \in X} \varphi_{k}(x)
$$

Due to Lemma 3.1 and inequality (3.13), Theorem 2.1 tells us that $c_{k}$ is a critical value of $\varphi_{k}$ and $c_{k} \geq \inf _{\tilde{H}_{k T}^{1}} \varphi_{k}$.

Inequality (3.11) tells us that $\inf _{\tilde{H}_{k T}^{1}} \varphi_{k}>-\infty$. Moreover, the definition of $c_{k}$ implies that $c_{k} \leq \sup _{x \in \mathbf{R}} \varphi_{k}\left(x+e_{k}\right)$. So, (3.12) holds.

Lemma 3.4 Under condition (B), we have

$$
\lim _{|x| \rightarrow+\infty} F(t,|x|+a)=-\infty \quad \forall t \in[0, T]
$$


Proof The main idea comes from [9]. For $|x|>0$, employing the mean value theorem for integrals, we have $F(t,|x|+a)=\int_{0}^{|x|} f(t, s+a) \mathrm{d} s=\int_{0}^{\frac{|x|}{2}} f(t, s+a) \mathrm{d} s+\int_{\frac{|x|}{2}}^{|x|} f(t, s+a) \mathrm{d} s \leq$ $\int_{\frac{|x|}{2}}^{|x|} f(t, s+a) \mathrm{d} s=f(t, \xi+a) \frac{|x|}{2}, \xi \in\left(\frac{|x|}{2},|x|\right)$. Then

$$
\limsup _{|x| \rightarrow+\infty} F(t,|x|+a) \leq \limsup _{|x| \rightarrow+\infty} f(t, \xi+a) \frac{|x|}{2}=-\infty \quad \forall t \in[0, T]
$$

which implies that (3.14) holds.

Lemma 3.5 If (3.14) holds, then for any constant $\delta>0$ there is a measurable subset $A_{\delta} \subset$ $[0, T]$ with meas $\left([0, T] \backslash A_{\delta}\right)<\delta$ such that

$$
F(t,|x|+a) \rightarrow-\infty, \quad \text { uniformly in } t \in A_{\delta}, \text { as }|x| \rightarrow+\infty
$$

Proof Set $f_{n}(t)=\inf _{|x|>n}-F(t,|x|+a)$. Then (3.14) implies that $f_{n}(t) \rightarrow+\infty$, as $n \rightarrow \infty$, $\forall t \in[0, T]$. By the continuity of $F(t, x)$ in $x$ and the measurability of $F(t, x)$ in $t$, for any $\delta>0$, by [12, Lemma 1], there exists a measurable subset $A_{\delta} \subset[0, T]$ with meas $([0, T] \backslash$ $\left.A_{\delta}\right)<\delta$ such that $f_{n}(t) \rightarrow+\infty$ as $n \rightarrow \infty$ uniformly for every $t \in A_{\delta}$. Then we obtain that $F(t,|x|+a) \rightarrow-\infty$, uniformly in $t \in A_{\delta}$, as $|x| \rightarrow+\infty$.

Lemma 3.6 For every $k \in \mathbf{N}^{*}$, let $x_{k}$ be a critical point of functional $\varphi_{k}$, then $\left\|x_{k}\right\|_{L^{\infty}} \rightarrow+\infty$ as $k \rightarrow+\infty$, if condition (f) holds.

Proof From conditions (f), (h1) and [9, (1) of Lemma 2.3], we have

$$
\begin{aligned}
\frac{c_{k}}{k} & =\frac{\varphi_{k}\left(x_{k}\right)}{k} \\
& \geq \frac{1}{k} \int_{0}^{k T} F\left(t,\left|x_{k}(t)\right|+a\right) \mathrm{d} t \\
& =\frac{1}{k} \int_{0}^{k T} \mathrm{~d} t \int_{0}^{\left|x_{k}(t)\right|} f(t, s+a) \mathrm{d} s \\
& \geq-\frac{1}{k} \int_{0}^{k T} \mathrm{~d} t \int_{0}^{\left|x_{k}(t)\right|}[\gamma(t) h(s)+g(t)] \mathrm{d} s \\
& \geq-\frac{1}{k} \int_{0}^{k T}\left[\gamma(t) h\left(\left\|x_{k}\right\|_{L^{\infty}}\right)+C \gamma(t)+g(t)\right]\left(\left\|x_{k}\right\|_{L^{\infty}}\right) \mathrm{d} t \\
& \geq-\left[\varepsilon\|\gamma\|_{L^{1}}\left\|x_{k}\right\|_{L^{\infty}}^{2}+\left(C_{\varepsilon}+C\right)\|\gamma\|_{L^{1}}\left\|x_{k}\right\|_{\left.L^{\infty}+\|g\|_{L^{1}}\left\|x_{k}\right\|_{L^{\infty}}\right]}\right. \\
& \geq-\left[\varepsilon C_{13}^{2}\|\gamma\|_{L^{1}}+C_{13}\left(C_{\varepsilon}+C\right)\|\gamma\|_{L^{1}}+C_{13}\|g\|_{L^{1}}\right]:=L .
\end{aligned}
$$

By (3.12), (3.15) and Lemma 3.5, the results in [9] and [12] imply that $\left\|x_{k}\right\|_{L^{\infty}} \rightarrow+\infty$ as $k \rightarrow+\infty$.

Proposition 3.2 Suppose $f$ satisfies conditions (B), (F2) and $x_{k}$ is a kT-periodic solution of (1.6), where $k \in \mathbf{N}^{*}$ is large enough, then $W_{k}^{1}=\left\{t \in \mathbf{R} \mid x_{k}(t)=0\right\}$ is nonempty and its points are isolated. Moreover, there exists at least one $t_{0} \in W_{k}^{1}$ such that $\dot{x}_{k}\left(t_{0}^{-}\right) \neq 0$. 
Proof The idea comes from [7, 9] and [11].

For every $k \in \mathbf{N}^{*}$, let $G_{k}(t)=\frac{1}{2}\left|\dot{x}_{k}(t)\right|^{2}-F\left(t,\left|x_{k}(t)\right|+a\right)$, then $G_{k}(t)$ is well-defined for all $t \in \mathbf{R}$ via (1.5), $G_{k}(t) \geq 0$ holds for all $t \in \mathbf{R}$ via the nonpositivity of $F$ and $G(t)$ is $k T$ periodic, continuous, differentiable for $t \in \mathbf{R} \backslash W_{k}^{1}$. By the definition of $G_{k}$, we get

$$
\begin{aligned}
G_{k}^{\prime}(t) & =\dot{x}_{k}(t)\left[\ddot{x}_{k}(t)-f\left(t,\left|x_{k}(t)\right|+a\right) \operatorname{sgn}\left(x_{k}(t)\right)\right]-\frac{\partial F\left(t,\left|x_{k}(t)\right|+a\right)}{\partial t} \\
& =-\frac{\partial F\left(t,\left|x_{k}(t)\right|+a\right)}{\partial t}, \quad t \in \mathbf{R} \backslash W_{k}^{1},
\end{aligned}
$$

then by (F2) we have

$$
\left|G_{k}^{\prime}(t)\right| \leq-\sigma F\left(t,\left|x_{k}(t)\right|+a\right) \leq \sigma G_{k}(t), \quad t \in \mathbf{R} \backslash W_{k}^{1} .
$$

If $G_{k}(0)=0$, then by Gronwall's inequality in [4], we have $G_{k}(t) \equiv 0$ on $\mathbf{R}$. Then $\dot{x}_{k}(t) \equiv 0$ and $F\left(t,\left|x_{k}(t)\right|+a\right) \equiv 0$ on $\mathbf{R}$, which means that $x_{k}(t) \equiv a_{k} \in \mathbf{R}$ and $f\left(t,\left|a_{k}\right|+a\right)=\ddot{x}_{k}(t) \equiv 0$, and so we get $\lim _{k \rightarrow+\infty} f\left(t,\left|a_{k}\right|+a\right)=0$. Lemma 3.6 implies that $\lim _{k \rightarrow+\infty}\left|a_{k}\right|=+\infty$, so we have $\lim _{\left|a_{k}\right| \rightarrow+\infty} f\left(t,\left|a_{k}\right|+a\right)=0$, which contradicts condition (B). Therefore we have $G_{k}(0)>0$.

Using (3.16), we get

$$
\left[G_{k}(t) \mathrm{e}^{\sigma t}\right]^{\prime}=\left(G_{k}^{\prime}(t)+\sigma G_{k}(t)\right) \mathrm{e}^{\sigma t} \geq 0, \quad t \in \mathbf{R} \backslash W_{k}^{1} .
$$

Note that $G_{k}(t) \mathrm{e}^{\sigma t}$ is continuous and (3.17) implies that $G_{k}(t) \mathrm{e}^{\sigma t} \geq G_{k}(0)$ holds for all $t \in$ $[0, k T]$, that is,

$$
G_{k}(t) \geq G_{k}(0) \mathrm{e}^{-\sigma t}>0, \quad t \in[0, k T] .
$$

To prove that $W_{k}^{1}$ is nonempty and discrete, it is sufficient to prove that $\widehat{W}_{k}^{1}=\{t \in$ $\left.[0, k T] \mid x_{k}(t)=0\right\}$ is nonempty and finite.

First, we consider whether $\widehat{W}_{k}^{1}$ is nonempty. If not, we have $x_{k} \in C^{2}([0, k T], \mathbf{R})$, and, without loss of generality, we suppose that $x_{k}(t)>0$ holds for all $t \in[0, k T]$. From (1.6), we get

$$
\ddot{x}_{k}(t)=f\left(t, x_{k}(t)+a\right) \quad \forall t \in[0, k T]
$$

and $x_{k}(t+k T)=x_{k}(t)$. By integrating the above equation on $[0, k T]$, we get

$$
0=\int_{0}^{k T} \ddot{x}_{k}(t) \mathrm{d} t=\int_{0}^{k T} f\left(t, x_{k}(t)+a\right) \mathrm{d} t
$$

which implies that $\ddot{x}_{k}(t)=f\left(t, x_{k}(t)+a\right) \equiv 0$ holds for any $t \in[0, k T]$ due to the nonpositivity of $f$. Since $x_{k}(t)$ is $k T$-periodic and continuous on $\mathbf{R}$, we have $x_{k}(t) \equiv b_{k}>0$ for all $t \in[0, k T]$. Lemma 3.6 implies that $\lim _{k \rightarrow+\infty} b_{k}=+\infty$. Then $\lim _{b_{k} \rightarrow+\infty} f\left(t, b_{k}+a\right)=0$, which contradicts condition (B). So $\widehat{W}_{k}^{1}$ is nonempty.

Next, we prove that the set $\widehat{W}_{k}^{1}$ is finite. Otherwise, since $\widehat{W}_{k}^{1}$ is compact, there would be a sequence $\left\{t_{j}\right\}_{1}^{\infty} \subset \widehat{W}_{k}^{1}$ with $0 \leq t_{1}<t_{2}<\cdots<t_{j}<\cdots \leq k T$, which, passing to a subsequence, still denoted by $\left\{t_{j}\right\}$, if necessary, we can take to be such that $t_{j} \rightarrow \beta \in \widehat{W}_{k}^{1}$ as 
$j \rightarrow+\infty$. Then

$$
\dot{x}_{k}\left(\beta^{-}\right)=\lim _{j \rightarrow+\infty} \frac{x_{k}\left(t_{j}\right)-x_{k}(\beta)}{t_{j}-\beta}=0 .
$$

On the other hand, since $\widehat{W}_{k}^{1}$ is nonempty, by (3.18), one has that $\dot{x}_{k}\left(t^{-}\right) \neq 0$ holds for all $t \in \widehat{W}_{k}^{1}$, which contradicts (3.19), so $\widehat{W}_{k}^{1}$ is finite.

Proof of Theorem 1.1 Proposition 3.1 tells us that $\varphi_{k}$ has a critical point $x_{k}$, and Lemma 2.2 implies that $x_{k}$ is a solution of (1.6) for every $k \in \mathbf{N}^{*}$. Proposition 3.2 implies that $u_{k}:=\left|x_{k}\right|$ satisfies Definition 1.1 for $k \in \mathbf{N}^{*}$ large enough, so $u_{k}$ is a nontrivial $k T$-periodic bouncing solution of system (1.4). Furthermore, Lemma 3.6 implies that $\left\|u_{k}\right\|_{L^{\infty}} \rightarrow+\infty$ as $k \rightarrow+\infty$. Thus we complete the proof.

\section{Example}

In this section, we present an example to demonstrate our Theorem 1.1.

Example 4.1 We define a $T$-periodic function $\theta \in C(\mathbf{R},(-\infty, 0))$ with

$$
\theta(t)= \begin{cases}-1, & t \in\left[0, \frac{T}{2}\right] \\ \sin \frac{2 \pi t}{T}-1, & t \in\left(\frac{T}{2}, T\right]\end{cases}
$$

Function $f: \mathbf{R} \times[a,+\infty) \rightarrow \mathbf{R}$ is defined as

$$
f(t, x)=\theta(t) \frac{2(x-a) \ln \left(100+(x-a)^{2}\right)-\frac{2(x-a)^{3}}{100+(x-a)^{2}}}{\ln ^{2}\left(100+(x-a)^{2}\right)}-1
$$

Then $f(t, x)$ is $T$-periodic in $t$, continuous for any $t$ and $x$, differentiable at every $t \in \mathbf{R}$ except at $t=\frac{m T}{2}\left(m \in \mathbf{N}^{*}\right)$ and

$$
F(t, x):=\int_{a}^{x} f(t, s) \mathrm{d} s=\theta(t) \frac{(x-a)^{2}}{\ln \left(100+(x-a)^{2}\right)}-(x-a) .
$$

The definition of function $f$ implies

$$
\begin{aligned}
|f(t,|x|+a)| & \leq|\theta(t)|\left[\frac{2|x|}{\ln \left(100+x^{2}\right)}+\frac{2|x|}{\ln ^{2}\left(100+x^{2}\right)}\right]+1 \\
& \leq 4|\theta(t)| \frac{|x|}{\ln \left(100+x^{2}\right)}+1, \quad \forall x \in \mathbf{R} \text { and } t \in[0, T] .
\end{aligned}
$$

Let $h(t)=\frac{t}{\ln \left(100+t^{2}\right)}, \gamma(t)=4|\theta(t)|$ and $g(t) \equiv 1$. Obviously, $h$ satisfies conditions (h1), (h2) and (h4). The results of [9] imply that $h$ satisfies condition (h3). So (4.1) implies that assumption (f) holds.

Note that $f(t, x+a)$ is the same as $f(t, x)$ in [9]. Similarly to paper [9], assumptions (F1), (F2) and (B) hold. 
Funding

This work was supported by National Natural Science Foundation of China [11371276, 10901118] and Elite Scholar Program in Tianjin University, P.R. China.

Availability of data and materials

Not applicable.

\section{Competing interests}

The authors declare that they have no competing interests.

\section{Authors' contributions}

The authors declare that the study was realized in collaboration with the same responsibility. All authors read and approved the final manuscript.

\section{Publisher's Note}

Springer Nature remains neutral with regard to jurisdictional claims in published maps and institutional affiliations.

Received: 26 April 2018 Accepted: 11 March 2019 Published online: 18 March 2019

\section{References}

1. Chang, K.C.: Variational methods for non-differentiable functionals and their applications to partial differential equations. J. Math. Anal. Appl. 80, 102-129 (1981). https://doi.org/10.1016/0022-247X(81)90095-0

2. Clarke, F.H.: A new approach to Lagrange multipliers. Math. Oper. Res. 1, 165-174 (1976). https://doi.org/10.1287/moor.1.2.165

3. Ding, W.: Subharmonic solutions of sublinear second order systems with impacts. J. Math. Anal. Appl. 379, 538-548 (2011). https://doi.org/10.1016/j.jmaa.2011.01.036

4. Evans, L.C.: Partial Differential Equations. Grad. Stud. Math., vol. 19, pp. 624-625. Am. Math. Soc., Rhode Island (1998)

5. Gasinski, L., Papageorgiou, N.S.: Nonsmooth Critical Point Theory and Nonlinear Boundary Value Problems. Ser. Math. Anal. Appl., pp. 181-183. Chapman \& Hall/CRC, Boca Raton (2005)

6. Gilbarg, D., Trudinger, N.S.: Elliptic Partial Differential Equations of Second Order. Springer, Berlin (2001)

7. Jiang, M.Y.: Periodic solutions of second order differential equations with an obstacle. Nonlinearity $19,1165-1183$ (2006) http://iopscience.iop.org/0951-7715/19/5/007

8. Mawhin, J., Willem, M.: Critical Point Theory and Hamiltonian Systems. Appl. Math. Sci., pp. 8-10. Springer, New York (1989)

9. Nie, Q.Q., Guo, F., Wang, M.W.: Generalized nonsmooth saddle point theorem and its applications on second order Hamiltonian systems. Chaos Solitons Fractals 104, 741-747 (2017). https://doi.org/10.1016/j.chaos.2017.09.032

10. Ning, Y., An, T.Q.: Periodic solutions of a class of nonautonomous second-order Hamiltonian systems with nonsmooth potentials. Bound. Value Probl. 2015, 34 (2015). http://link.springer.com/article/10.1186/s13661-015-0292-x

11. Qian, D.B., Torres, P.J.: Periodic motions of linear impact oscillators via the successor map. SIAM J. Math. Anal. 36, 1707-1725 (2005). https://doi.org/10.1137/S003614100343771X

12. Tang, C.L., Wu, X.P.: Periodic solutions for second order systems with not uniformly coercive potential. J. Math. Anal. Appl. 259, 386-397 (2001). https://doi.org/10.1006/jmaa.2000.7401

\section{Submit your manuscript to a SpringerOpen ${ }^{\circ}$ journal and benefit from:}

- Convenient online submission

- Rigorous peer review

- Open access: articles freely available online

- High visibility within the field

Retaining the copyright to your article

Submit your next manuscript at $\boldsymbol{~ s p r i n g e r o p e n . c o m ~}$ 